\title{
SUMMABILITY FACTORS FOR CESÀRO METHODS
}

\author{
DAVID F. DAWSON
}

ABstract. It is shown that if each of $r$ and $s$ is a nonnegative integer and $\left\{f_{p}\right\}$ is a complex sequence such that $\Sigma f_{p} a_{p}$ is Cesaro summable of order $s$ whenever $\Sigma a_{p}$ is Cesaro summable of order $r$, then $\Sigma f_{p} a_{p}$ is Cesàro summable of order $r$ whenever $\Sigma a_{p}$ is Cesàro summable of order $r$.

In [2, p. 310] Tetsuzo Kojima obtained the following result.

THEOREM K. In order for $\left\{f_{p}\right\}$ to have the property that $\sum f_{p} a_{p}$ is Cesàro summable of order $s((C, s)$-summable $)$ whenever $\Sigma a_{p}$ is $(C, r)$-summable, it is necessary and sufficient that the following conditions hold:

$$
\sup _{n} n^{r-s}\left|f_{n}\right|<\infty
$$

and

$$
\sup _{n} \frac{1}{A_{n}^{(s)}} \sum_{i=1}^{n-r-1} A_{i}^{(r)}\left|\sum_{k=0}^{r+1}\left(\begin{array}{c}
r+1 \\
k
\end{array}\right) A_{n-i+1}^{(s-k)} \Delta^{r-(k-1)} f_{i+k}\right|<\infty
$$

where $A_{n}^{(k)}=(n+k-1) ! / k !(n-1) !, A_{n}^{(-k)}=0, n, k=1,2,3, \ldots$, and each of $r$ and $s$ is a nonnegative integer.

In the present paper, we simplify Kojima's conditions for certain cases, and, as a consequence, are able to see a surprising phenomenon: If $\sum f_{p} a_{p}$ is $(C, s)$-summable whenever $\sum a_{p}$ is $(C, r)$-summable, then $\sum f_{p} a_{p}$ is $(C, r)$ summable whenever $\Sigma a_{p}$ is $(C, r)$-summable.

To simplify Kojima's conditions, we note first that, for $i$ fixed,

$$
\lim _{n} \frac{A_{n-i+1}^{(s-k)}}{A_{n}^{(s)}}=\lim _{n} \frac{\left(\begin{array}{cc}
n-i+1+s-k-1 \\
s-k
\end{array}\right)}{\left(\begin{array}{c}
n+s-1 \\
s
\end{array}\right)}= \begin{cases}0 & \text { if } k>0 \\
1 & \text { if } k=0\end{cases}
$$

Note that (*) holds even if $s-k<0$, since the numerator of the first

Received by the editors June 27, 1978.

AMS (MOS) subject classifications (1970). Primary 40G05, 40D15.

Key words and phrases. Cesaro summability, bounded variation, summability factors. 
fraction is $\mathbf{0}$ for this case. Using (*) we find that, for $i$ fixed,

$$
\begin{aligned}
\lim _{n}\left[\frac{A_{i}^{(r)}}{A_{n}^{(s)}}\right. & \left.\sum_{k=0}^{r+1}\left(\begin{array}{c}
r+1 \\
k
\end{array}\right) A_{n-i+1}^{(s-k)} \Delta^{r-(k-1)} f_{i+k}\right] \\
& =\sum_{k=0}^{r+1}\left[\left(\begin{array}{c}
r+1 \\
k
\end{array}\right) A_{i}^{(r)} \Delta^{r-(k-1)} f_{i+k} \lim _{n} \frac{A_{n-i+1}^{(s-k)}}{A_{n}^{(s)}}\right] \\
& =A_{i}^{(r)} \Delta^{r+1} f_{i} \\
& =\left(\begin{array}{c}
i+r-1 \\
r
\end{array}\right) \Delta^{r+1} f_{i} .
\end{aligned}
$$

Thus if (2) holds, then

$$
\sum_{i=1}^{\infty}\left(\begin{array}{c}
i+\underset{r}{r-1} \\
r
\end{array}\right)\left|\Delta^{r+1} f_{i}\right|<\infty .
$$

Note the special case of $\left(2^{\prime}\right)$ when $r=0$ :

$$
\sum_{i=1}^{\infty}\left|\Delta f_{i}\right|<\infty
$$

which means that $f=\left\{f_{p}\right\}$ is of bounded variation, and consequently is not only bounded but convergent.

Next we show that for $k<r$ and $1<i<n$,

$$
\frac{A_{i}^{(r)} A_{n-i+1}^{(s-k)}}{A_{n}^{(s)}} \leqslant(s !)(r !) A_{i+k}^{(r-k)} .
$$

We note that (**) holds if $s<k$, since $A_{n-i+1}^{(s-k)}=0$ for this case. For $2<i<n$ and $0<k<\min \{s, r\}$, we have

$$
\begin{aligned}
\frac{A_{i}^{(r)} A_{n-i+1}^{(s-k)}}{A_{n}^{(s)}}= & \frac{(i+r-1) !}{r !(i-1) !} \frac{(n-i+1+s-k-1) !}{(s-k) !(n-i+1-1) !} / \frac{(n+s-1) !}{s !(n-1) !} \\
= & \frac{[(i+k-1) \cdots(i)][(n-1) \cdots(n-i+1)]}{(n+s-1)(n+s-2) \cdots(n+s-i-k+1)} \\
& \cdot \frac{s !(r-k) !}{r !(s-k) !} \cdot \frac{(i+r-1) !}{(r-k) !(i+k-1) !} \\
\leqslant & \frac{(n+k-1)(n+k-2) \cdots(n-i+1)}{(n+s-1)(n+s-2) \cdots(n+s-i-k+1)} \\
& \cdot(s !)(r !)\left(\begin{array}{c}
i+r-1 \\
r-k
\end{array}\right) \\
< & (s !)(r !) A_{i+k}^{(r-k)} .
\end{aligned}
$$

We omit similar proofs for the two cases $k=0, i>1$ and $k>0, i=1$.

Now suppose (1) and (2') hold and $s<r$. Then $f \in \mathrm{BV}_{r+1}$, where $\mathrm{BV}_{j}$ is 
the set of all bounded complex sequences $x$ such that

$$
\sum_{p=1}^{\infty}\left(\begin{array}{c}
p+j-2 \\
j-1
\end{array}\right)\left|\Delta^{j} x_{p}\right|<\infty
$$

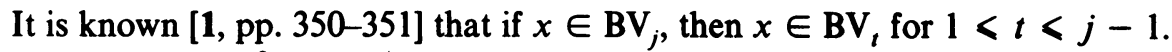
Thus $\sum_{p=1}^{\infty}\left(\begin{array}{c}p+t-2 \\ t-1\end{array}\right)\left|\Delta^{t} f_{p}\right|<\infty$ for $1<t<r+1$, so that, using (**), we have

$$
\begin{aligned}
\frac{1}{A_{n}^{(s)}} & \sum_{i=1}^{n-r-1} A_{i}^{(r)}\left|\sum_{k=0}^{r+1}\left(\begin{array}{c}
r+1 \\
k
\end{array}\right) A_{n-i+1}^{(s-k)} \Delta^{r-(k-1)} f_{i+k}\right| \\
& \leqslant \sum_{i=1}^{n-r-1} \sum_{k=0}^{r}\left(\begin{array}{c}
r+1 \\
k
\end{array}\right) \frac{A_{i}^{(r)} A_{n-i+1}^{(s-k)}}{A_{n}^{(s)}}\left|\Delta^{r-(k-1)} f_{i+k}\right| \\
& \leqslant \sum_{i=1}^{n-r-1} \sum_{k=0}^{r}\left(\begin{array}{c}
r+1 \\
k
\end{array}\right)(s !)(r !) A_{i+k}^{(r-k)}\left|\Delta^{r-(k-1)} f_{i+k}\right| \\
& \leqslant(r+1) !(r !)(s !) \sum_{i=1}^{n-r-1} \sum_{k=0}^{r}\left(\begin{array}{c}
i+k+r-k-1 \\
r-k
\end{array}\right)\left|\Delta^{r-(k-1)} f_{i+k}\right| \\
& \leqslant(r+1) !(r !)(s !) \sum_{k=0}^{r} \sum_{i=1}^{\infty}\left(\begin{array}{c}
i+r-1 \\
r-k
\end{array}\right)\left|\Delta^{r-k+1} f_{i+k}\right|
\end{aligned}
$$

and consequently, since the last expression is free of $n$, we see that (2) holds.

Thus we have shown that if (1) and ( $\left.2^{\prime}\right)$ hold and $s<r$, then (2) holds, and we previously showed that (2) implies $\left(2^{\prime}\right)$ in any case. Hence we have the following result.

THEOREM 1. In order for $f$ to have the property that $\sum f_{p} a_{p}$ is $(C, s)$-summable whenever $\Sigma a_{p}$ is $(C, r)$-summable, $s<r$, it is necessary and sufficient that (1) and $\left(2^{\prime}\right)$ hold.

The following special case of Theorem 1 is especially useful for our purpose:

In order for $f$ to have the property that $\sum f_{p} a_{p}$ is $(C, r)$-summable whenever $\sum a_{p}$ is $(C, r)$-summable, it is necessary and sufficient that $f \in \mathrm{BV}_{r+1}$.

THEOREM 2. If $\sum f_{p} a_{p}$ is $(C, s)$-summable whenever $\sum a_{p}$ is $(C, r)$-summable, then $\sum f_{p} a_{p}$ is $(C, r)$-summable whenever $\sum a_{p}$ is $(C, r)$-summable.

Proof. The statement is obvious if $s<r$. Suppose $r<s$. From Theorem K and a previous argument, we see that $\left(2^{\prime}\right)$ holds. Now since the set of convergent sequences $((C, 0)$-summable sequences) is a subset of the set of $(C, r)$-summable sequences, we know that $\sum f_{p} a_{p}$ is $(C, s)$-summable whenever $\Sigma a_{p}$ is $(C, 0)$-summable. Hence from Theorem $\mathrm{K}$ and a previous argument, $\left(2^{\prime \prime}\right)$ holds, so that $f$ is bounded. But $f$ bounded and (2') imply that $f \in \mathrm{BV}_{r+1}$, and this, by the special case of Theorem 1 mentioned above, means that $\sum f_{p} a_{p}$ is $(C, r)$-summable whenever $\sum a_{p}$ is $(C, r)$-summable. 
D. F. DAWSON

\section{REFERENCES}

1. D. F. Dawson, A generalization of a theorem of Hans Hahn concerning matrix summability, Boll. Un. Mat. Ital. (4) 3 (1970), 349-356.

2. Tetsuzo Kojima, On generalized Toeplitz's theorems on limit and their applications, Tôhoku Math. J. 12 (1917), 291-326.

Departmint of Mathematics, North Texas State Universtry, Denton, Texas 76203 